\title{
Radio Detection of Cosmic Rays with LOFAR
}

\author{
J.R. Hörandel ${ }^{* 1,2}$, S. Buitink ${ }^{3}$, A. Corstanje ${ }^{1}$, J.E. Enriquez ${ }^{1}$, H. Falcke ${ }^{1,2,4}$, \\ T. Karskens ${ }^{1}$, M. Krause ${ }^{1,5}$, A. Nelles ${ }^{1,6}$, J.P. Rachen ${ }^{1}$, L. Rossetto ${ }^{1}$, P. Schellart ${ }^{1}$, \\ O. Scholten ${ }^{7,8}$, S. ter Veen ${ }^{1,4}$, S. Thoudam ${ }^{1}$, T.N.G. Trinh ${ }^{7}$
}

1 Department of Astrophysics/IMAPP, Radboud University Nijmegen, P.O. Box 9010, 6500 GL

Nijmegen, The Netherlands

2 NIKHEF, Science Park Amsterdam, 1098 XG Amsterdam, The Netherlands

3 Astrophysical Institute, Vrije Universiteit Brussel, Pleinlaan 2, 1050 Brussels, Belgium

4 Netherlands Institute of Radio Astronomy (ASTRON), Postbus 2, 7990 AA Dwingeloo, The

Netherlands

5 Now at: DESY, Platanenallee 6, 15738 Zeuthen, Germany

6 Now at: Department of Physics and Astronomy, University of California Irvine, Irvine, CA

92697-4575, USA

7 KVI-CART, University Groningen, P.O. Box 72, 9700 AB Groningen, The Netherlands

8 Interuniversity Institute for High-Energy, Vrije Universiteit Brussel, Pleinlaan 2, 1050

Brussels, Belgium

E-mail: j.horandeleastro.ru.nl

\begin{abstract}
When high-energy cosmic rays (ionized atomic nuclei) impinge on the atmosphere of the Earth they interact with atomic nuclei and initiate cascades of secondary particles - the extensive air showers. Many of the secondary particles in the air showers are electrons and positrons. They cause radiation in the frequency range of tens of MHz. The LOFAR radio telescope detects this radiation in the frequency range 30 to $240 \mathrm{MHz}$. LOFAR has a high antenna density and good time resolution. In turn, the properties of the radio emission are measured in detail. The properties of the shower-inducing cosmic rays are derived from the air shower measurements, namely their direction, energy, and particle type (atomic mass). The uncertainties achieved are competative to established techniques. This demonstrates that the radio technique is now a standard tool to measure extensive air showers and to study the properties of the incoming cosmic rays. The mean logarithmic mass of cosmic rays as measured with LOFAR is derived as a function of energy. In an examplary study, these data are used to show that the radio measurements of air showers are now in a state to discriminate astrophysical models of the origin of cosmic rays.
\end{abstract}

The 34th International Cosmic Ray Conference,

30 July- 6 August, 2015

The Hague, The Netherlands

\footnotetext{
* Speaker.
} 

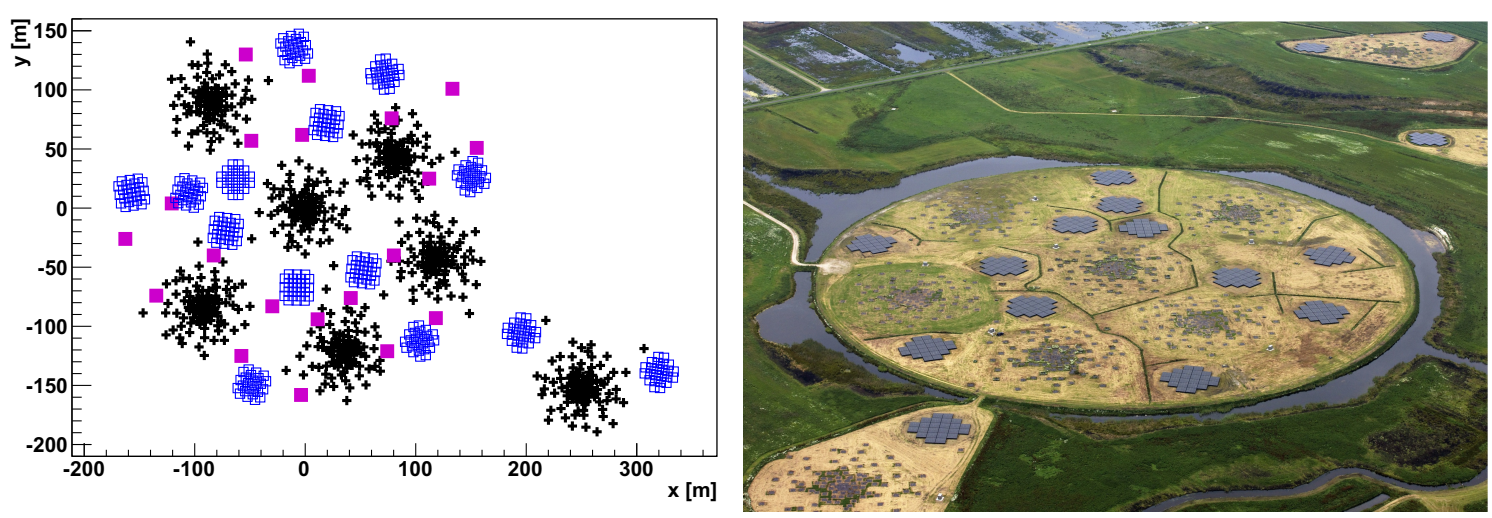

Figure 1: The center of LOFAR [10,11]. Left: The six stations to the left form the Superterp. The crosses indicate the low-band antennas (black). The open squares (blue) show the positions of the high-band antenna tiles, which are split into two groups per station. The filled squares (pink) indicate the positions of the LORA particle detectors. Right: Aerial photograph.

\section{Introduction}

To understand the origin of high-energy cosmic rays is one of the open key questions in astroparticle physics [1, 2]. An inspiring article, published in 2003 [3] accompanied the renaissance of radio detection of extensive air showers with the ultimate goal to measure the properties of cosmic rays with this technique and the pioneer experiments LOPES [4] and CODALEMA [5] have been initiated. The big success of these pathfinders stimulated further investigations of the radio emission of air showers on larger scales, with installations such as Tunka-Rex [6], AERA at the Pierre Auger Observatory [7, 8], and the LOFAR radio telescope. Significant progress has been achieved in the last decade [9] and we now understand the emission processes of the radio waves in the atmosphere. Most of the emission is due to the interaction of the shower with the magnetic field of the Earth, which leads to an transverse current in the shower. In addition to this emission, the overabundance of electrons in the shower that are collected from atmospheric molecules leads to a current in the direction of the shower.

\section{LOFAR Radio Telescope}

A modern radio detector for extensive air showers is the LOFAR radio telescope (in particular, its dense core in the Netherlands). The layout of the LOFAR core is depicted in Fig. 1. The LOFAR key science project Cosmic Rays represents one of the six scientific key objectives of LOFAR $[10,11]$. LOFAR is a digital radio telescope. Its antennas are spread over several European countries and are used together for interferometric radio observations in the frequency range of $10-240 \mathrm{MHz}$. The density of antennas increases towards the center of LOFAR. Here, about 2400 antennas are clustered on an area of roughly $10 \mathrm{~km}^{2}$. This high density of antennas together with the excellent time resolution makes LOFAR the perfect tool to study features of the radio emission created by extensive air showers. Air shower measurements are conducted based on a trigger received from an array of scintillators (LORA) [12, 13], which results in a read-out of the ring buffers that store the raw voltage traces per antenna for up to $5 \mathrm{~s}$. LOFAR comprises two types 

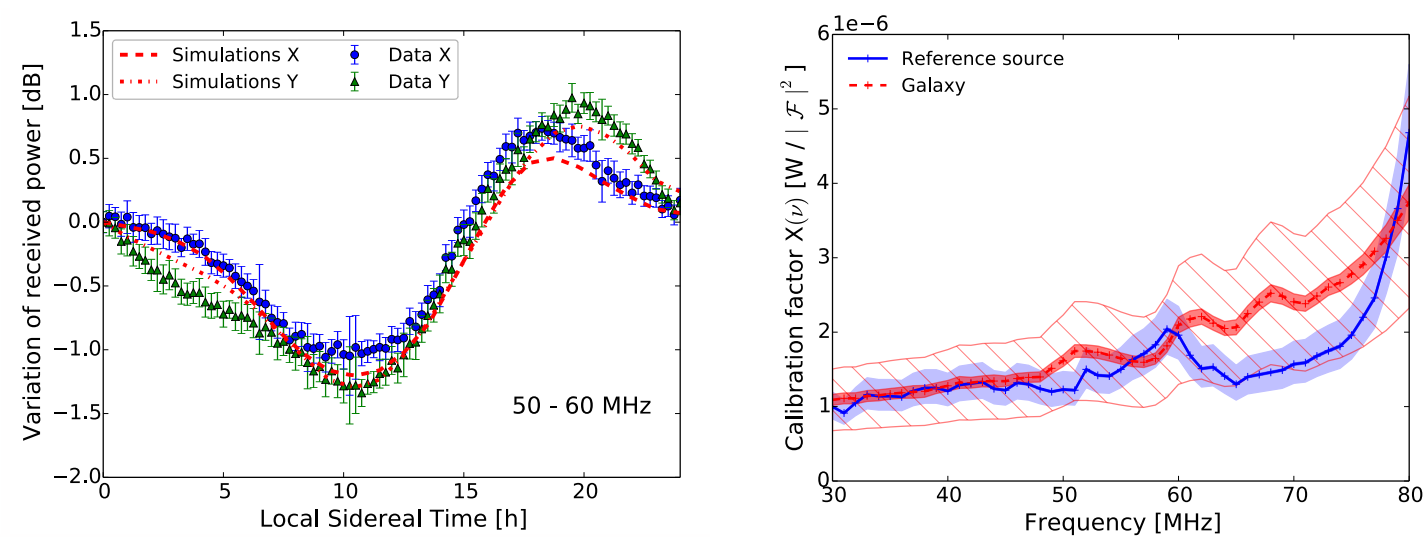

Figure 2: Left: Integrated median noise power as a function of the Local Sidereal Time, for the $50-60 \mathrm{MHz}$ sub-band. Also shown is the predicted received power in both dipoles (dashed red lines) after applying electronic noise corrections. For details see [14]. Right: Calibration factors as function of frequency across the LOFAR band for the Galactic and the reference-source calibrations. Both calibration curves contain statistical uncertainties of the method in the dark region, with systematic uncertainties illustrated by the lighter region (dashed for Galactic, filled for reference source).

of antennas, recording radio emission in low-frequency band from 10 to $90 \mathrm{MHz}$ and also in the high-frequency band $(110-240 \mathrm{MHz})$.

The LOFAR Radboud Air Shower Array - LORA is comprised of 20 scintillation counters distributed over the area of the inner core of LOFAR, see Fig. 1. The all-particle energy spectrum of cosmic rays has been measured with this set-up in the energy range from about $10^{16}$ to about $10^{18} \mathrm{eV}$. The measurements from the scintillator array are used to establish an absolute energy scale for the air-shower measurements [13].

Several campaigns have been conducted to calibrate the radio antennas in situ with different reference sources [14]. In particular, the reference sources used to calibrate LOPES and Tunka-Rex as well as AERA have been used at LOFAR. Thus, a direct cross calibration has been performed between these major radio air-shower detectors. Three approaches were used to check and improve the antenna model of LOFAR and to provide an absolute calibration of the whole system for air shower measurements. Two methods are based on calibrated reference sources and one on a calibration approach using the diffuse radio emission of the Galaxy, optimized for short data-sets. An accuracy of $19 \%$ in amplitude is reached.

For illustration some results are shown in Fig. 2. The figure (left) shows the variation of the received power (in the frequency band $50-60 \mathrm{MHz}$ ) as a function of the local siderial time. The recorded galactic emission exhibits the expected siderial modulation. By comparing the recorded intensity to expectations from a galactic emission model, an absolute calibration is obtained as a function frequency, as illustreted in the figure (right). The calibration function thus obtained is in excellent agreement with a calibration using a calibrated reference source located about $12 \mathrm{~m}$ above a LOFAR antenna in the field. 

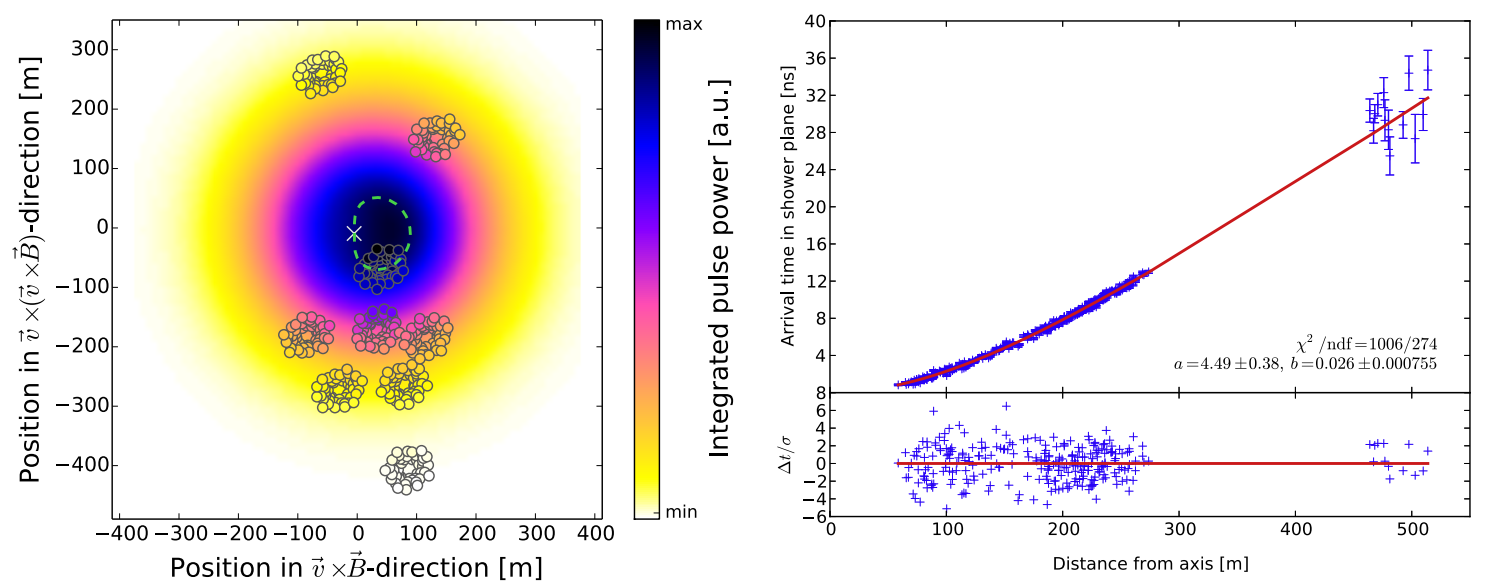

Figure 3: Left: Footprint of an air shower measured with LOFAR The colored background represents predictions of the radio signal according to simulations [15]. Right: The arrival time of the signals, relative to a plane as a function to the distance to the shower axis as measured with LOFAR. The lower right graph illustrates the arrival time differences with respect to a fit (hyperboloid) [16].

\section{Precision measurement of the radio emission in air showers}

LOFAR combines a high antenna density and a fast sampling of the measured voltage traces in each antenna. This yields very detailed information for each measured air shower and the properties of the radio emission have been measured with high precision. Important aspects of radio emission in air showers are reviewed in the following. We focus on radio emission in the frequency range $30-80 \mathrm{MHz}$, only one result (Fig. 4 right) deals with higher frequencies.

Lateral distribution function of the radio signals The footprint of the radio emission recorded at ground level is not rotationally symmetric $[15,17,18]$, such as e.g. the particle content of a shower, see Fig. 3 (left). Radio emission is generated through interactions with the Earth magnetic field, which yield a bean-shaped footprint on the ground. The correct reference system is in the shower plane (perpendicular to the shower axis), with one coordinate axis along the $\vec{v} \times \vec{B}$ direction and the second axis along the $\vec{v} \times(\vec{v} \times \vec{B})$ direction. Where $\vec{v}$ is the propagation velocity vector of the shower (parallel to the shower axis) and $\vec{B}$ represents the direction and strength of the Earth magnetic field. The measured power in the frequency range $30-80 \mathrm{MHz}$ is plotted as a function of the distance to the shower axis in Fig. 4 (left). For example at a distance of $200 \mathrm{~m}$ from the shower axis, ambiguities are visible in this one-dimensional projection: the recorded signal strength is a function of the azimuth angle, which results in the visible structure. Historically, the lateral distribution of the radio signal on the ground has often be parameterized with a simple exponential function (e.g. [19]). However, the LOFAR measurements suggest that the radio emission should be parameterized by a more complex expression: a two-dimensional Gaussian function is used to describe the approximately exponential fall-off at large distances form the shower axis. A second (smaller) two-dimensional Gaussian function is subtracted from the first one to describe the ring structure of the signal close to the shower axis. To reproduce the observed bean shape, the centers of both Gaussian functions are slightly offset. The power at position $\left(x^{\prime}, y^{\prime}\right)$ in the shower plane 

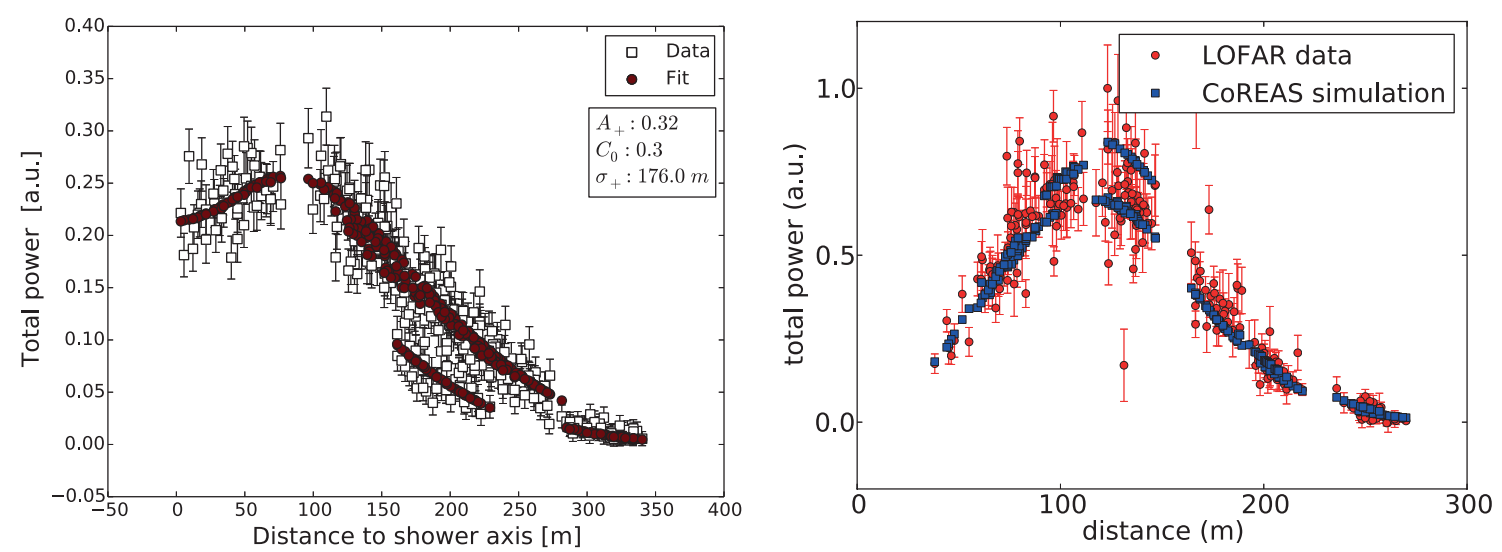

Figure 4: Total power measured in an air shower as a function of the distance to the shower axis (in the shower plane) as measured by LOFAR in the frequency range $30-80 \mathrm{MHz}$ (left) [17] and $110-190 \mathrm{MHz}$ (right) [20].

(perpendicular to the shower axis) is described as

$$
P\left(x^{\prime}, y^{\prime}\right)=A_{+} \exp \left(-\frac{\left(x^{\prime}-X_{+}\right)^{2}+\left(y^{\prime}-Y_{+}\right)^{2}}{\sigma_{+}^{2}}\right)-A_{-} \exp \left(-\frac{\left(x^{\prime}-X_{-}\right)^{2}+\left(y^{\prime}-Y_{-}\right)^{2}}{\sigma_{-}^{2}}\right),
$$

The parameters are the scaling factors $A_{+}$and $A_{-}$(where in general $A_{-}>0$ ), the width of the Gaussian functions $\sigma_{+}$and $\sigma_{-}$, and the centers of the two-dimensional Gaussian distributions $\left(X_{+}, Y_{+}\right)$ and $\left(X_{-}, Y_{-}\right)$.

First quantitative measurements in the frequency range 120-240 $\mathrm{MHz}$ Radio emission from extensive air showers has also been recorded with the LOFAR high-band antennas in the $200 \mathrm{MHz}$ frequency domain [20]. The measured power is depicted in Fig. 4 (right) as a function of the distance to the shower axis. A clear maximum is visible at distances around $120 \mathrm{~m}$ in this onedimensional projection, indicating a clear (Čerenkov-like) ring structure. Such rings are predicted from theory [21]: relativistic time compression effects lead to a ring of amplified emission, which starts to dominate the emission pattern for frequencies above $\sim 100 \mathrm{MHz}$. The LOFAR data clearly confirm the importance to include the index of refraction of air as a function of height into calculations of the radio emission.

Shape of the shower front The precise shape of the radio wave front is a long-standing issue. In the literature different scenarios have been discussed: a spherical, conical, or hyperbolical shape (e.g. [22]). The LOFAR findings clearly indicate that a hyperboloid is the best way to describe the shape of the measured wave front [16]. A hyperboloid asymptotically reaches a conical shape at large distances from the shower axis and can be approximated as a sphere close to the shower axis. A measured wave front of a shower registered with LOFAR is shown in Fig. 3 (right). The time difference relative to a plane is plotted as a function of a distance to the shower axis. The line indicates a fit of a hyperboloid to the measured data. The lower part of the graph shows the time differences of the individual antennas with respect to the fit function. 

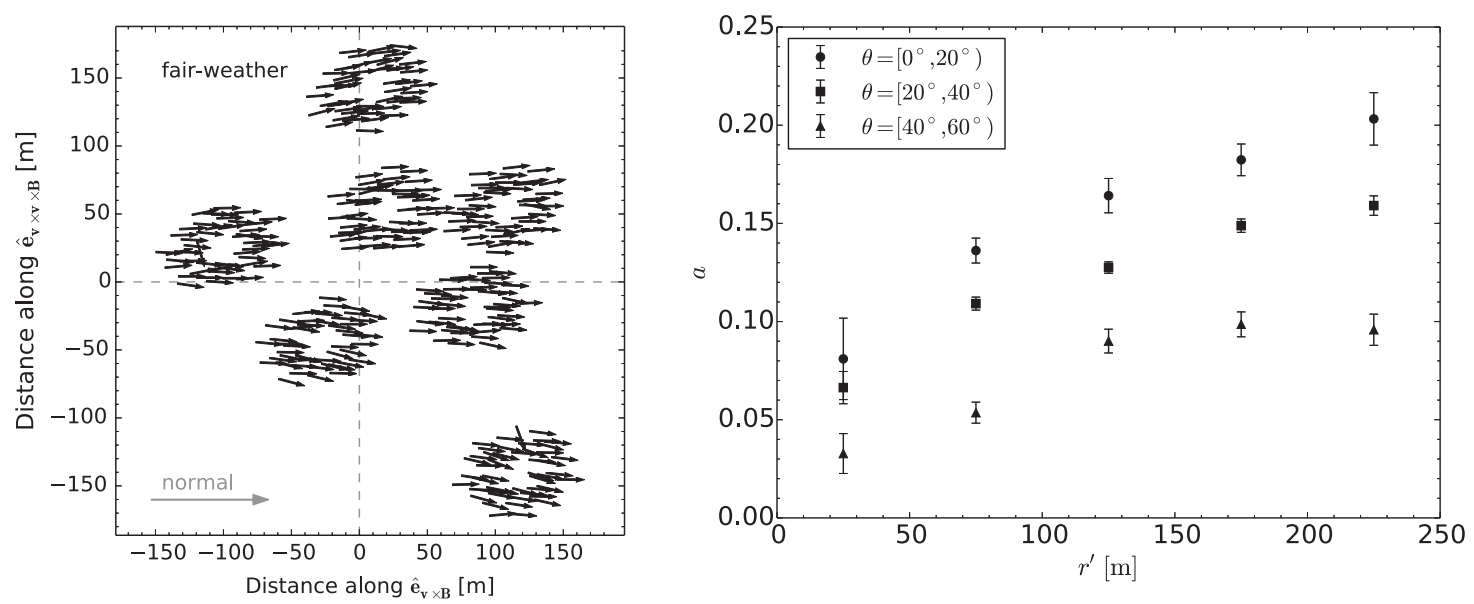

Figure 5: Left: Polarization as measured with individual LOFAR antennas (arrows) in the shower plane during fair weather conditions. The expected polarization direction for fair-weather air showers is indicated with "normal". The position of the shower axis, orthogonal to the shower plane, is indicated by the intersection of the dashed lines [23]. Right: The ratio $a$ between contributions due to the Askaryan effect and the geomagnetic emission is plotted and as a function of the distance to the shower axis (in the shower plane) for showers with different zenith angles as measured by LOFAR (right) [24].

Polarization of the radio signal The radio emission in extensive air showers originates from different processes. The dominant mechanism is of geomagnetic origin [4, 25]. Electrons and positrons in the shower are accelerated in opposite directions by the Lorentz force exerted by the magnetic field of the Earth. The generated radio emission is linearly polarized in the direction of the Lorentz force $(\vec{v} \times \vec{B})$, where $\vec{v}$ is the propagation velocity vector of the shower (parallel to the shower axis) and $\vec{B}$ represents the direction and strength of the Earth magnetic field. A secondary contribution to the radio emission results from the excess of electrons at the front of the shower (Askaryan effect) [26, 27]. This excess is built up from electrons that are knocked out of atmospheric molecules by interactions with shower particles and by a net depletion of positrons due to annihilation. This charge excess contribution is radially polarized, pointing towards the shower axis. The resulting emission measured at the ground is the sum of both components. Interference between these components may be constructive or destructive, depending on the position of the observer/antenna relative to the shower. The emission is strongly beamed in the forward direction due to the relativistic velocities of the particles. Additionally, the emission propagates through the atmosphere, which has a non-unity index of refraction that changes with height. This gives rise to relativistic time-compression effects, most prominently resulting in a ring of amplified emission around the Čerenkov angle, see Fig. 4. By precisely measuring the polarization direction of the electric field at each antenna position (see Fig. 5 left), the ratio $a=\varepsilon_{\text {Askaryan }} / \varepsilon_{\text {geom }}$ between the contributions from the Askaryan effect and the geomagnetic emission is measured. At LOFAR the ratio $a$ has been measured as a function of the distance to the shower axis for showers with different zenith angles, as depicted in Fig. 5 (right) [24]. The figure illustrates that the contribution through the Askaryan effect increases with increasing distance to the shower axis and it is more pronounced for vertical showers (with small zenith angle). The values range from a contribution of less than a 

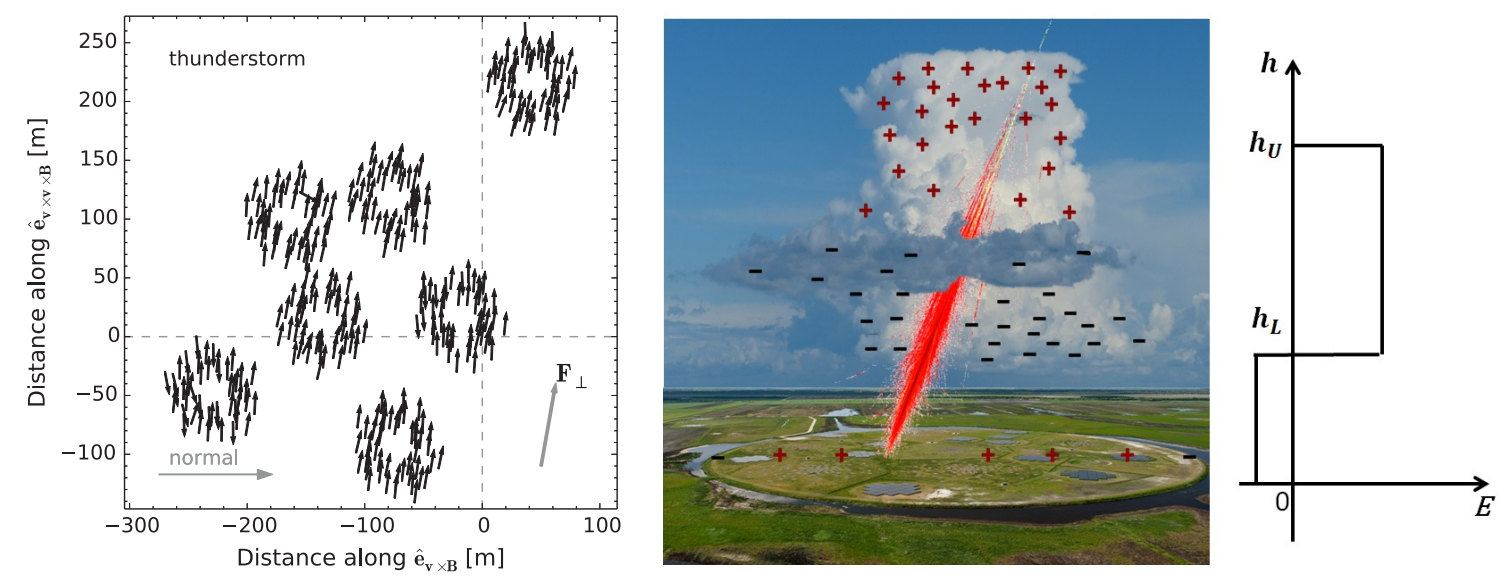

Figure 6: Left: Polarization as measured with individual LOFAR antennas (arrows) in the shower plane during thunderstorm conditions. The expected polarization direction for fair-weather air showers is indicated with "normal". The position of the shower axis, orthogonal to the shower plane, is indicated by the intersection of the dashed lines [23]. See also Fig. 5 (left) for comparison. Right: A schematic structure of a thundercloud is given where charge is accumulated at the bottom and the top layer. An air shower (in red) is passing through the thundercloud. The LOFAR core is seen as a circular structure on the ground. The structure of the induced electric field is given schematically on the right hand side [30].

few $\%$ for horizontal showers, close to the shower axis to values above $20 \%$ for vertical showers at a distance of $250 \mathrm{~m}$ from the shower axis. The obtained values are in good agreement with an average value $a=14 \% \pm 2 \%$ as obtained at the Pierre Auger Observatory [28].

Confirmation of simulation codes The detailed investigations of the properties of the radio emission and the comparison between measurements and predictions from simulations demonstrate that the simulation code CoREAS [29] fully describes all relevant features of the radio emission in air showers and the predictions can be used to interpret the measured air shower data.

Probing atmospheric electric fields during thunderstorms Radio detection of air showers is also used for auxiliary science, such as the measurements of electric fields in the atmosphere during thunderstorms $[31,32,23]$. The footprint of the radio emission from an air shower, which developed during a thunderstorm is shown in Fig. 6 (left) [23]. The intensity and polarization patterns of such air showers are radically different from those measured during fair-weather conditions, as shown in Fig. 5 (left). The figures illustrate the polarization as measured with individual LOFAR antennas (arrows) in the shower plane. LOFAR antennas are grouped into circular stations, of which seven are depicted. An arrow labeled "normal" indicates the expected polarization direction for fair weather conditions. The position of the shower axis, orthogonal to the shower plane, is indicated by the intersection of the dashed lines. A simple two-layer model for the atmospheric electric field is used as illustrated in Fig. 6 (right). Parameters of the model are the heights of the boundaries of the electric fields $h_{l}$ and $h_{u}$ and the electric field strengths $E_{l}$ and $E_{u}$ in the lower and upper layer, respectively. For the shower shown in the figure the parameters obtained are $h_{l}=2.9 \mathrm{~km}, h_{u}=8 \mathrm{~km},\left|E_{u}\right|=50 \mathrm{kV} / \mathrm{m}$, and $\left|E_{l}\right| /\left|E_{u}\right|=0.53$. With this model the measured 

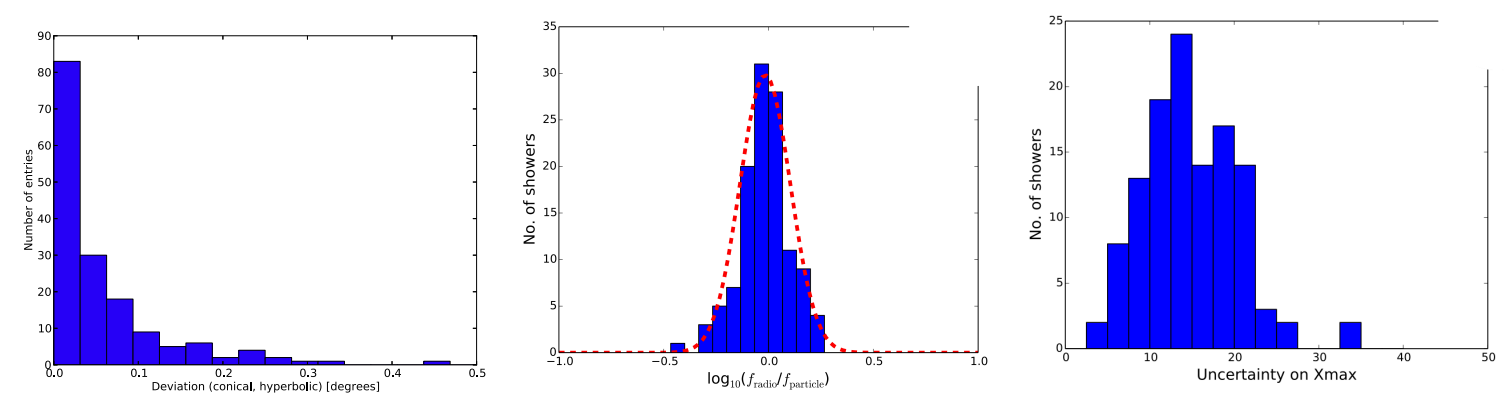

Figure 7: Resolution to measure the shower parameters with the radio technique. Left: Angular difference between the reconstructed shower axais direction, describing the wavefront with a conical and a hyperbolic wave front, typical values are below $0.1^{\circ}$ [16]. Center: Energy resolution with an average of about $32 \%$. Right: Resolution for the depth of the shower maximum $X_{\max }$ with an average of about $17 \mathrm{~g} / \mathrm{cm}^{2}[37,38]$.

patterns are well reproduced by state-of-the-art simulation codes. This in turn provides a novel way to study atmospheric electric fields.

\section{Measuring properties of cosmic rays with the radio technique}

Ultimate objective is to fully characterize the incoming cosmic ray with the radio measurements, i.e. to determine its arrival direction, its energy, and the particle type/mass (see e.g. [33, 18, $34,35,36])$.

Arrival direction A precise description of the shape of the shower front is essential to correctly reconstruct the direction of the incoming cosmic ray. Different shapes have been investigated at LOFAR as discussed above [16]. Reconstructing the arrival directions of the same measured showers with different wavefront shapes results in differences in the reconstructed arrival directions of typically less than $1^{\circ}$ between a plane and a hyperboloid and typically less than $0.1^{\circ}$ between a cone and a hyperboloid, see Fig. 7 (left). The figure shows the anglular difference, typical values are below $0.1^{\circ}$. Based on these investigations it is expected that the angular resolution for the arrival direction of the shower is better than $1^{\circ}$.

Energy The parameters of the function to model the intensity pattern of the radiation on the ground (as described above) are sensitive to the properties of the shower-inducing cosmic rays [17]. The integral of the measured power density is proportional to the shower energy. This is illustrated in Fig. 8 (left). The shower energy is plotted as a function of a parameter, being proportional to the integrated power. The measured signal strength is corrected for a factor, which depends on the angle between the shower axis and the direction of the geomagnetic field. This is necessary, since the dominating geomagnetic emission strongly depends on this angle. The shower energy is determined in two ways in the figure: it is derived from Monte Carlo simulations (using CORSIKA [39] and CoREAS [29]) and it has been measured with the particle detector array at LOFAR (LORA). A clear correlation is seen between the shower energy and the measured radio intensity on the ground. 

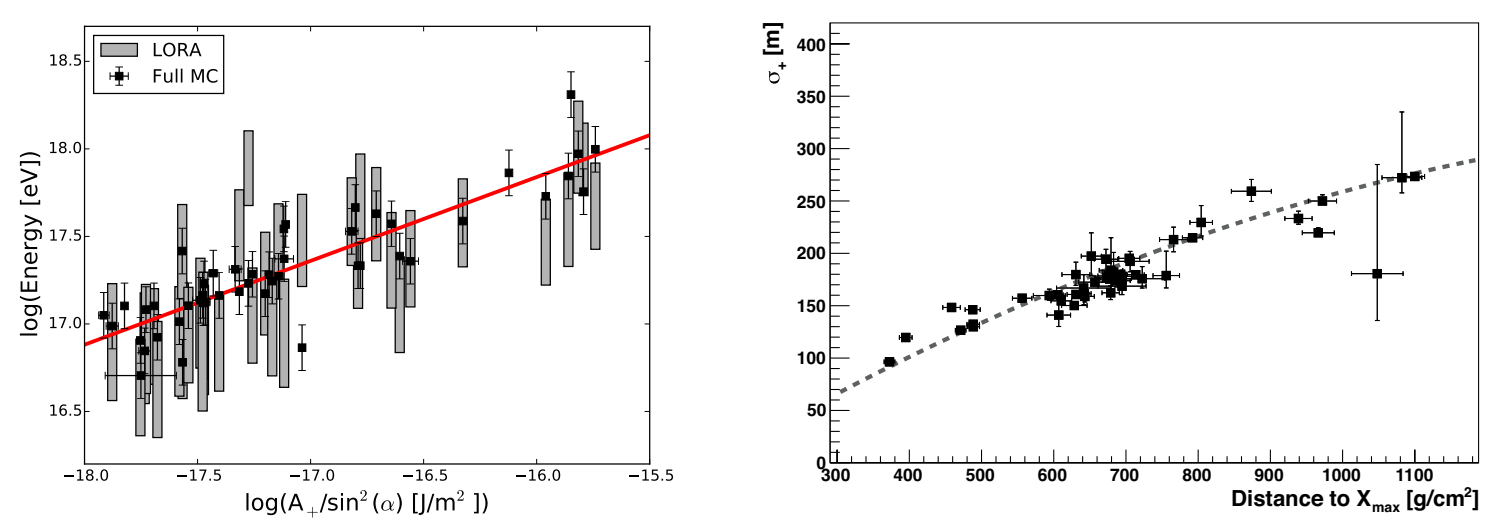

Figure 8: Determining the properties of cosmic rays with radio measurements [17]. Left: Energy of the cosmic ray (determined from simulations and measured with the LORA air shower array) as a function of a quantity, being in principle proportional to the integrated measured radio power. Right: Width of the footprint of the radio emission as a function of the distance to the shower maximum.

Depth of the shower maximum The most challenging task is to derive the type of the incoming cosmic ray or its atomic mass $A$ from the radio measurements. Key is to measure the depth of the shower maximum $X_{\max }$ in the atmosphere, which is proportional to $\ln A$. The width of the measured footprint is proportional to the geometrical distance from the antennas to the position of the shower maximum. This correlation is shown in Fig. 8 (right). This method is used at LOFAR [17] (and has been adapted to the Auger Observatory [18]) to estimate the cosmic-ray mass.

To obtain a precise value of the depth of the shower maximum for each shower, we apply the following procedure [40, 37]. The shower direction and energy are obtained from the measured signals in the particle detectors and the radio antennas. With these parameters simulations are initiated, simulating the development of air showers and the accompanied radio emission, using the CORSIKA and the CoREAS codes, for primary protons and iron nuclei. Due to fluctuations in the individual particle interactions in an air shower this results in showers with a wide distribution for the depth of the shower maximum (all showers having the same energy and direction of incidence as the measured shower). The predicted signals in the particle detectors and the radio antennas are then compared to the measurements. A $\chi^{2}$ method is used to determine the simulated shower, which best matches the measured values. This yields a value for the depth of the shower maximum. The method is illustrated in Fig. 9 (left). The $\chi^{2}$ values are depicted as a function of the depth of the shower maximum $X_{\max }$. A parabola is fit to the $\chi^{2}$ values and the minimum of this function gives the estimated $X_{\max }$ value for the measured shower. With this method $X_{\max }$ is determined with an accuracy of about $17 \mathrm{~g} / \mathrm{cm}^{2}$ at LOFAR, see Fig. 7 (right), where the uncertainty in $X_{\max }$ is shown for individual showers. During this procedure the energies of the showers are slightly scaled in order to best match the measured signals in the radio antennas and the particle detectors. For the same shower, the energies obviously should be identical. The difference between these scaling factors is taken as the energy resolution. An average value of about $32 \%$ is obtained, as depicted in Fig. 7 (center).

With this method the average depth of the shower maximum has been measured for three 

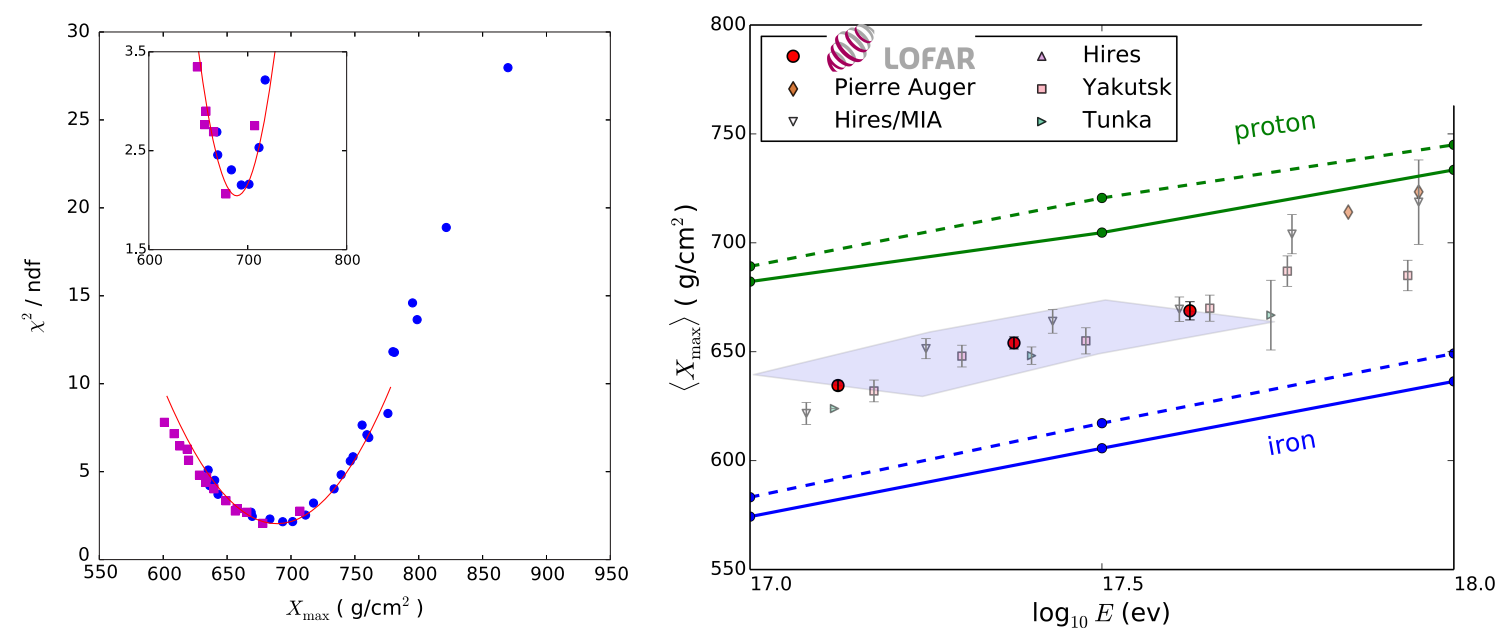

Figure 9: Left: Determining the depth of the shower maximum from the radio measurements. The $\chi^{2}$ values of simulated showers are shown as a function of the depth of the shower maximum [40]. Right: Average depth of the shower maximum as measured with LOFAR [40, 37, 38]. The results are compared to other measurements from the literature, for details and references, see [37, 38]. Also shown are predictions for protons (green) and iron nuclei (blue), according to the hadronic interaction models EPOS-LHC (solid line) and QGSJET-II-4 (dashed line).

energy bins around energies of $10^{17}$ to $10^{17.6} \mathrm{eV}$ as shown in Fig. 9 (right) [37, 38]. The LOFAR measurements are compared to results from the literature. The lines indicate predictions according to the hadronic interaction models EPOS [41] and QGSJET [42, 43] for protons and iron nuclei.

The relative distance between the data points and the predictions for protons and iron nuclei is a measure for the mean logarithmic mass of the cosmic rays (see e.g. [44] for a detailed discussion)

$$
\langle\ln A\rangle \equiv \sum_{i} r_{i} \ln A_{i}=\frac{X_{\max }^{\text {meas }}-X_{\max }^{p}}{X_{\max }^{F e}-X_{\max }^{p}} \cdot \ln A_{F e} .
$$

$r_{i}$ are the relative fractions of elements with mass $A_{i}$ in cosmic rays. $X_{\max }^{\text {meas }}$ are the measured values and $X_{\max }^{p}$ and $X_{\max }^{F e}$ are predictions for protons and iron nuclei at a given energy, respectively. Converting the LOFAR measurements yields values around $\ln A \approx 1.9$ to 2.4 at energies above $10^{17} \mathrm{eV}$

\section{Potential of the radio technique}

The mass measurements of LOFAR are in an astrophysically very interesting energy range between $10^{17}$ and $10^{18} \mathrm{eV}$, where a transition is expected from a galactic to an extragalactic origin of cosmic rays (e.g. [1, 46]). The values obtained are depicted in Fig. 10 as a function of energy. The LOFAR values (interpreted with two hadronic interaction models) are compared to values from the literature (for details and references, see [45]). The difference between the interpretations with the different hadronic interaction models is of the order of $\Delta \ln A \approx 0.4$ and illustrates the systematic uncertainty introduced by the limited understanding of inelastic hadronic interactions at these energies. 


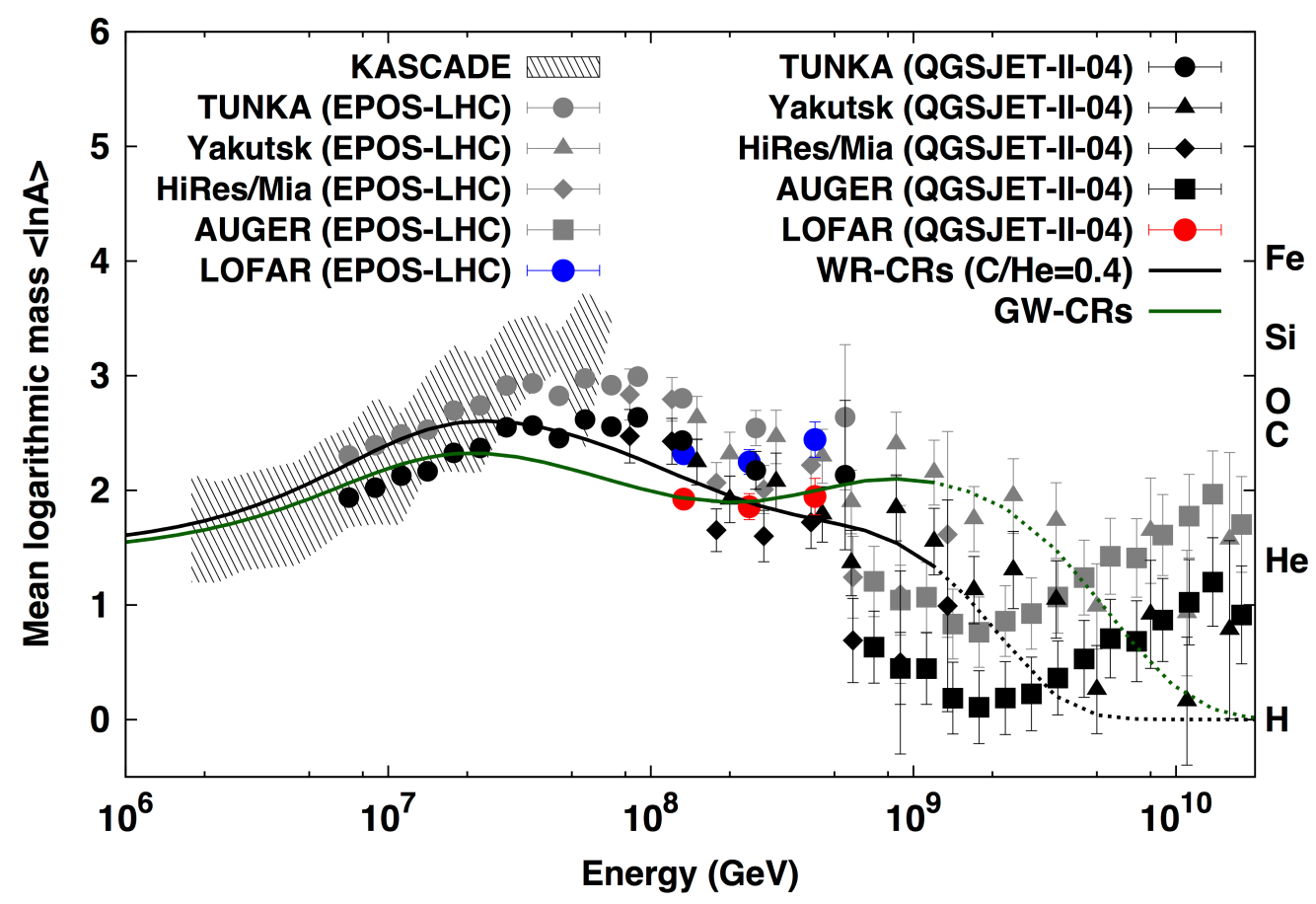

Figure 10: Mean logarithmic mass $\langle\ln A\rangle$ of cosmic rays as a function of energy [45]. Results from LOFAR as shown in Fig. 9 are compared to data from the literature. The lines illustrate predictions of two models to describe the origin of cosmic rays. For details and references, see [45].

A general trend can be seen in the figure: a rise of the mean logarithmic mass as a function of energy between $10^{15}$ and almost $10^{17} \mathrm{eV}$ as expected due to the subsequent fall-off of individual elements in Galactic cosmic rays as a function of rigidity $(E / Z)$ towards the end of the Galactic component (e.g. [47, 48]). The mean $\ln A$ decreases as a function of energy in the energy range between roughly $10^{17}$ and $10^{18} \mathrm{eV}$, in this region the extragalactic cosmic-ray component contributes more and more to the observed measured cosmic-ray (all-particle) flux. At the highest energies $\left(E>10^{18} \mathrm{eV}\right)$ again an icnrease of $\langle\ln A\rangle$ is observed as a function of energy.

The lines represent predictions of two recent models to describe the origin of cosmic rays [45]. Motivated by the recent high-precision measurements of the cosmic-ray energy spectrum and mass composition by several new-generation experiments, a detailed study has been conducted to understand the observed properties of (Galactic) cosmic rays up to about $10^{18} \mathrm{eV}$. The study involves building a propagation model for cosmic rays, originating from supernova explosions in the interstellar medium. Although these cosmic rays can satisfactorily explain the observed spectra of different elements at low energies, provided by balloon and satellite-borne experiments, it has been found that they cannot account completely for the cosmic rays above $\approx 10^{16} \mathrm{eV}$ observed by air shower experiments. An additional component of Galactic cosmic rays is required in order to explain the observed cosmic rays beyond this energy up to about $10^{18} \mathrm{eV}$. Two cases have been studied: cosmic rays being re-accelerated in the galactic wind ("GW-CRs", green curve in the figure) and a special class of supernovae, originating from Wolf-Rayet stars, being able to effectively accelerate cosmic rays at high energies ("WR-CRs", black line in the figure). As can be inferred 
from the figure, both models describe the mass composition quite well. In this two-component model, the knee and the second knee in the all-particle energy spectrum of cosmic rays are caused by the cut-offs in the energy spectra of the first and the second component, respectively. The first component also explains the spectral breaks observed in the proton and helium spectra at about $200 \mathrm{GeV} /$ nucleon.

Taking the LOFAR QGSJET values literally would clearly prefer the "Galactic Wind" model. This illustrates that the radio measurements of air showers are now competitive and start to discriminate astrophysical models of the origin of cosmic rays.

\section{Conclusion and outlook}

Radio emission from extensive air showers in the frequency range from 30 to $80 \mathrm{MHz}$ is now routinely observed with several experimental set-ups. The results of CODALEMA, LOPES, Tunka-Rex, AERA and, in particular, the very detailed results of LOFAR, with its high density of antennas and the excellent time resolution, have provided a wealth of information to understand the emission processes. They are now well understood and well described by latest air shower simulation codes such as e.g. CORSIKA together with CoReas.

With LOFAR it has been demonstrated that the relevant inmformation about the showerinducing primary cosmic ray can be measured with the radio technique: namely its direction, its energy and its particle type (mass $A$ of nucleus). Uncertainties are competative to established techniques, i.e. better than $1^{\circ}$ for the direction, about $30 \%$ energy resolution, and a resolution for $X_{\max }$ better than $20 \mathrm{~g} / \mathrm{cm}^{2}$.

LOFAR (and also Tunka-Rex and AERA at the Pierre Auger Observatory) measure in the very interesting energy range from $10^{17}$ to $10^{18} \mathrm{eV}$. In the next years, precise measurements of the mass composition of cosmic rays are expected from these facilities. They will significantly contribute to our understanding of the origin of cosmic rays in this energy range.

\section{References}

[1] J. Blümer, R. Engel, and J. Hörandel, Cosmic rays from the knee to the highest energies, Prog. Part. Nucl. Phys. 63 (2009) 293.

[2] M. Nagano and A. Watson, Observations and implications of the ultrahigh-energy cosmic rays, Rev. Mod. Phys. 72 (2000) 689.

[3] H. Falcke and P. Gorham, Detecting radio emission from cosmic ray air showers and neutrinos with a digital radio telescope, Astropart. Phys. 19 (2003) 477.

[4] H. Falcke et al., Detection and imaging of atmospheric radio flashes from cosmic ray air showers, Nature 435 (2005) 313.

[5] D. Ardouin et al., Radio-detection signature of high energy cosmic rays by the CODALEMA experiment, Nucl. Instr. \& Meth. A 555 (2005) 148.

[6] D. Kostunin, et al., Tunka-Rex: Status and results of the first measurements, Nucl.Instrum.Meth. A742 (2014) 89.

[7] P. Abreu et al., Antennas for the Detection of Radio Emission Pulses from Cosmic-Ray, JINST 7 (2012) P10011. 
[8] P. Abreu et al., Advanced functionality for radio analysis in the Offline software framework of the Pierre Auger Observatory, Nucl.Instrum.Meth. A635 (2011) 92.

[9] T. Huege, The renaissance of radio detection of cosmic rays, Braz.J.Phys. 44 (2014) 520.

[10] M. van Haarlem, et al., LOFAR: The LOw-Frequency ARray, Astronomy \& Astrophysics 556 (2013) A2.

[11] P. Schellart, et al., Detecting cosmic rays with the LOFAR radio telescope, Astron.Astrophys. 560 (2013) A98.

[12] S. Thoudam, et al., LORA: A scintillator array for LOFAR to measure extensive air showers, Nucl.Instrum.Meth. A767 (2014) 339.

[13] S. Thoudam et al., Measurement of the cosmic-ray energy spectrum above $10^{16} \mathrm{eV}$ with the LOFAR Radboud Air Shower Array, Astropart. Phys. 73 (2016) 34.

[14] A. Nelles et al., Calibrating the absolute amplitude scale for air showers measured at LOFAR, JINST 10 (2015), no. 11 P11005.

[15] A. Nelles, et al., A parameterization for the radio emission of air showers as predicted by CoREAS simulations and applied to LOFAR measurements, Astropart.Phys. 60 (2015) 13.

[16] A. Corstanje, et al., The shape of the radio wavefront of extensive air showers as measured with LOFAR, Astropart.Phys. 61 (2015) 22.

[17] A. Nelles, et al., The radio emission pattern of air showers as measured with LOFAR-a tool for the reconstruction of the energy and the shower maximum, JCAP 1505 (2015), no. 05018.

[18] J. Schulz, et al., Status and prospects of the Auger Engineering Radio Array, Proceedings 34th International Cosmic Ray Conference, Den Haag (2015) PoS(ICRC2015)615.

[19] H. Allan, Progress in Elementary Particles and Cosmic Ray Physics, p. 169. J.G. Wilson and S.G. Wouthuysen eds., North Holland, 1971.

[20] A. Nelles, et al., Measuring a Cherenkov ring in the radio emission from air showers at 110-190 $\mathrm{MHz}$ with LOFAR, Astropart.Phys. 65 (2014) 11.

[21] K. Werner and O. Scholten, Macroscopic treatment of radio emission from cosmic ray air showers based on shower simulations, Astropart. Phys. 29 (2008) 393.

[22] W. Apel, et al., The wavefront of the radio signal emitted by cosmic ray air showers, JCAP 1409 (2014), no. 09025.

[23] P. Schellart, et al., Probing Atmospheric Electric Fields in Thunderstorms through Radio Emission from Cosmic-Ray-Induced Air Showers, Phys.Rev.Lett. 114 (2015), no. 16165001.

[24] P. Schellart, et al., Polarized radio emission from extensive air showers measured with LOFAR, JCAP 1410 (2014), no. 10014.

[25] D. Ardouin et al., Geomagnetic origin of the radio emission from cosmic ray induced air showers observed by CODALEMA, Astropart. Phys. 31 (2009) 192.

[26] V. Marin, et al., Charge excess signature in the CODALEMA data. Interpretation with SELFAS2., in Proceedings, 32nd International Cosmic Ray Conference (ICRC 2011), vol. 1, pp. 291, 2011.

[27] A. Bellétoile, et al., Evidence for the charge-excess contribution in air shower radio emission observed by the CODALEMA experiment, Astropart.Phys. 69 (2015) 50. 
[28] A. Aab et al., Probing the radio emission from air showers with polarization measurements, Phys.Rev. D89 (2014), no. 5052002.

[29] T. Huege, M. Ludwig, and C. W. James, Simulating radio emission from air showers with coreas, ARENA 2012, AIP Conf. Proc. 1535 (2013) 128.

[30] T. N. G. Trinh et al., Influence of Atmospheric Electric Fields on the Radio Emission from Extensive Air Showers, Physical Review D (2016) in press.

[31] S. Buitink et al., Amplified radio emission from cosmic ray air showers in thunderstorms, Astron. \& Astroph. 467 (2007) 385.

[32] W. Apel, et al., Thunderstorm Observations by Air-Shower Radio Antenna Arrays, Adv.Space Res. 48 (2011) 1295.

[33] W. D. Apel et al., Reconstruction of the energy and depth of maximum of cosmic-ray air-showers from LOPES radio measurements, Phys. Rev. D90 (2014), no. 6062001.

[34] C. Glaser et al., Energy estimation for cosmic rays measured with the auger engineering radio array, AIP Conf. Proc. 1535 (2013) 68.

[35] C. Glaser et al., The energy content of extensive air showers in the radio frequency range of 30-80 mhz, Proceedings 34th International Cosmic Ray Conference, Den Haag (2015) PoS(ICRC2015)364.

[36] A. Aab et al., Energy Estimation of Cosmic Rays with the Engineering Radio Array of the Pierre Auger Observatory, Submitted to: Phys. Rev. D (arXiv 1508.04267) (2015) [arXiv: 1508 . 0426 ].

[37] S. Buitink et al., Measuring the cosmic ray mass composition with LOFAR, Proceedings 34th International Cosmic Ray Conference, Den Haag (2015) PoS(ICRC2015)368.

[38] S. Buitink et al. Nature (2016) in press.

[39] D. Heck et al., “Corsika: A Monte Carlo code to simulate extensive air showers.” Report FZKA 6019, Forschungszentrum Karlsruhe, 1998.

[40] S. Buitink, et al., Method for high precision reconstruction of air shower $X_{\text {max }}$ using two-dimensional radio intensity profiles, Phys.Rev. D90 (2014), no. 8082003.

[41] T. Pierog, I. Karpenko, J. M. Katzy, E. Yatsenko, and K. Werner, EPOS LHC: Test of collective hadronization with data measured at the CERN Large Hadron Collider, Phys. Rev. C92 (2015), no. 3 034906.

[42] S. Ostapchenko, QGSJET-II: physics, recent improvements, and results for air showers, EPJ Web Conf. 52 (2013) 02001.

[43] S. Ostapchenko, Monte Carlo treatment of hadronic interactions in enhanced Pomeron scheme: I. QGSJET-II model, Phys. Rev. $\mathbf{D 8 3}$ (2011) 014018.

[44] J. Hörandel, On total inelastic cross sections and the average depth of the maximum of extensive air showers, J. Phys. G: Nucl. Part. Phys. 29 (2003) 2439.

[45] S. Thoudam et al., A study of the energy spectrum and composition of cosmic rays up to the highest energies, Proceedings 34th International Cosmic Ray Conference, Den Haag (2015) PoS(ICRC2015)526.

[46] K.-H. Kampert and M. Unger, Measurements of the Cosmic Ray Composition with Air Shower Experiments, Astropart. Phys. 35 (2012) 660.

[47] J. Hörandel, On the knee in the energy spectrum of cosmic rays, Astropart. Phys. 19 (2003) 193.

[48] J. Hörandel, Models of the knee in the energy spectrum of cosmic rays, Astropart. Phys. 21 (2004) 241. 